\title{
BMJ Open How do middle-aged and older adults with chronic hip pain view their health problem and its care? A protocol for a systematic review and qualitative evidence synthesis
}

\author{
Travis Haber (D) , ${ }^{1}$ Rana S Hinman, ${ }^{1}$ Fiona Dobson, ${ }^{2}$ Samantha Bunzli, ${ }^{3}$ \\ Michelle Hall (D) ${ }^{1}$
}

To cite: Haber T, Hinman RS, Dobson F, et al. How do middle-aged and older adults with chronic hip pain view their health problem and its care? A protocol for a systematic review and qualitative evidence synthesis. BMJ Open 2021;11:e053084. doi:10.1136/ bmjopen-2021-053084

- Prepublication history and additional supplemental material for this paper are available online. To view these files, please visit the journal online (http://dx.doi.org/10.1136/ bmjopen-2021-053084).

Received 04 May 2021 Accepted 11 0ctober 2021

\section{Check for updates}

\section{(C) Author(s) (or their} employer(s)) 2021. Re-use permitted under CC BY-NC. No commercial re-use. See rights and permissions. Published by BMJ.

${ }^{1}$ Centre for Health, Exercise and Sports Medicine, Department of Physiotherapy, School of Health Sciences, The University of Melbourne, Melbourne, Victoria, Australia

${ }^{2}$ Physiotherapy, The University of Melbourne, Melbourne, Victoria, Australia

${ }^{3}$ Department of Surgery, St Vincent's Hospital, The University of Melbourne, Melbourne, Victoria, Australia

Correspondence to

Dr Michelle Hall;

halm@unimelb.edu.au

\section{ABSTRACT}

Introduction Chronic hip pain in middle-aged and older adults is common and disabling. Patient-centred care of chronic hip pain requires a comprehensive understanding of how people with chronic hip pain view their health problem and its care. This paper outlines a protocol to synthesise qualitative evidence of middle-aged and older adults' views, beliefs, expectations and preferences about their chronic hip pain and its care.

Methods and analysis We will perform a qualitative evidence synthesis using a framework approach. We will conduct this study in accord with the Preferred Reporting Items for Systematic Reviews and Meta-Analyses (PRISMA) Statement and the Enhancing Transparency in Reporting the synthesis of Qualitative research checklist. We will search MEDLINE, CINAHL, The Cochrane Central Register of Controlled Trials, EMBASE and PsycINFO using a comprehensive search strategy. A priori selection criteria include qualitative studies involving samples with a mean age over 45 and where $80 \%$ or more have chronic hip pain. Two or more reviewers will independently screen studies for eligibility, assess methodological strengths and limitations using the Critical Appraisal Skills Programme qualitative studies checklist, perform data extraction and synthesis and determine ratings of confidence in each review finding using the Grading of Recommendations Assessment, Development and Evaluation-Confidence in the Evidence from Reviews of Qualitative research approach. Data extraction and synthesis will be guided by the Common-Sense Model of Self-Regulation. All authors will contribute to interpreting, refining and finalising review findings. This protocol is registered on PROSPERO and reported according to the PRISMA Statement for Protocols (PRISMA-P) checklist.

Ethics and dissemination Ethics approval is not required for this systematic review as primary data will not be collected. The findings of the review will be disseminated through publication in an academic journal and scientific conferences.

PROSPERO registration number PROSPERO registration number: CRD42021246305.

\section{Strengths and limitations of this study}

- We will use a systematic approach guided by the Cochrane Qualitative and Implementation Methods Group, including search strategies, methods of data extraction and synthesis and choice of tools for quality appraisal (Critical Appraisal Skills Programme; CASP qualitative study checklist) and ratings of confidence (Grading of Recommendations Assessment, Development and Evaluation-Confidence in the Evidence from Reviews of Qualitative research; GRADE-CERQual approach).

- To minimise bias, at least two review authors will independently screen studies against a priori selection criteria, extract data using a custom-developed a priori template, conduct quality appraisal (CASP qualitative study checklist), synthesise data and generate ratings of confidence (GRADE-CERQual approach).

- Our study will be limited by excluding studies not published in English, which precludes transferring our findings to non-English-speaking countries or minorities.

- Our study will be further limited by relying on published data-potentially missing important details or meaning present in the unpublished data and increasing the risk of researcher bias.

\section{INTRODUCTION}

Chronic hip pain is common and disabling, ${ }^{1-7}$ particularly in middle age and beyond. The prevalence of chronic hip pain increases with age, affecting $14 \%-36 \%$ of people 56 years or older. ${ }^{5}{ }^{7}$ People with chronic hip pain typically experience reduced physical function, social isolation, emotional distress and reduced quality of life. ${ }^{1-48}$ Common diagnoses of chronic hip pain in middleaged or older adults include osteoarthritis, gluteal tendinopathy (also greater trochanter pain syndrome and lateral hip pain) and 
femoroacetabular impingement. ${ }^{2} 9{ }^{10}$ Hip conditions often coexist. $^{11}{ }^{12}$ Clinical guidelines recommend education, exercise, weight loss and analgesics as treatments for non-systemic hip pain..$^{210}{ }^{13-16}$ However, the use of recommended treatments and satisfaction with care is low among people with chronic hip pain. ${ }^{817-20}$ Enhancing healthcare through patient-centred approaches is a key priority of the WHO and national health organisations across the world. ${ }^{21-24}$

The WHO defines people-centred care as 'an approach to care that consciously adopts the perspectives of individuals, families and communities and sees them as participants as well as beneficiaries of trusted health systems that respond to their needs and preferences in humane and holistic ways' ${ }^{25}$ Patient-centred care improves patient satisfaction and adherence to care and treatment outcomes (including pain), while reducing costs, across a range of health settings and conditions, such as hospital care, joint replacements, musculoskeletal pain and osteoarthritis. ${ }^{26-38}$ While clinical guidelines for musculoskeletal pain recommend that care is patient centred, ${ }^{39}{ }^{40}$ people with joint pain often receive care that fails to address their beliefs, wants and needs. ${ }^{18} 41-43$

People with joint pain are often dissatisfied with healthcare $^{1842}$; they believe healthcare fails to address their pain and its psychosocial impact and fails to educate them about their pain and the treatments available to them. ${ }^{18} 4244-46$ Health professionals often inform or fail to address unhelpful patient beliefs, such as attributing pain to wear and tear. ${ }^{8} 4547$ Dissatisfactory healthcare and unhelpful patient beliefs deter people from using, or adhering to, recommended treatments, such as exercise. ${ }^{8184248}$ By better addressing a person's beliefs, wants and needs, patient-centred care could better guide people with chronic hip pain to helpful coping strategies. However, to deliver patient-centred care, we need a comprehensive understanding of the beliefs, wants and needs among people with chronic hip pain.

We identified four systematic reviews and two scoping reviews exploring peoples' views, experiences, beliefs and attitudes about osteoarthritis and/or its care-among people with osteoarthritis at different joints, including hip osteoarthritis. ${ }^{18} 4142454649$ Unhelpful beliefs were identified among people with osteoarthritis who delayed or avoided healthcare and exercised less. ${ }^{41454649}$ People with osteoarthritis were often dissatisfied with how clinicians communicated with them and with the information they provided. ${ }^{184245}$

Although insightful, there are limitations to the reviews conducted to date. The reviews are limited to people with osteoarthritis and predominantly isolated to knee osteo$\operatorname{arthritis}^{184142454649}$; it is, thus, unclear whether, and how, their findings apply to people with hip osteoarthritis, or to people whose hip pain may result from conditions other than osteoarthritis. Five of the six existing reviews did not evaluate how much confidence could be placed in the review findings. ${ }^{18} 41424649$ Without assessing the extent to which review findings reasonably represent the phenomenon of interest, it is unclear how useful the findings are for decision-making, implementing interventions and/or developing guidelines. ${ }^{50}$ Finally, only one of the six reviews used social or psychological theory to guide their methods or results. ${ }^{45}$ Social and psychological theories provide a lens to extract and interpret data from primary studies and to report review findings-helping to explain patient health behaviours, including healthcare use. $^{52-56}$ The Cochrane Qualitative and Implementation Methods Group recommend selecting theories that best capture the phenomenon of interest of the review and are empirically supported. ${ }^{54}$

The Common Sense Model of Self-Regulation (CSM) describes how people form beliefs about their health problems and then draw on these beliefs to choose coping strategies (eg, seeking care or avoiding activities). ${ }^{57-59}$ Beliefs about a health problem are informed by one's own and observed experiences, by what is heard in the wider public discourse (eg, the media), and by discussions with significant others and health professionals. ${ }^{57-60}$ People will update their beliefs about their health problem and what they should do about it-whether to maintain or modify their coping strategies-by judging whether their coping strategies are moving them towards or away from their goals. ${ }^{57-59}$ The CSM has been used to understand beliefs, coping responses and health outcomes among people with chronic hip pain, hip and knee osteoarthritis and back pain. ${ }^{875961-68}$ By using the CSM to guide our data synthesis, we can better understand and explain how peoples' beliefs impact how they cope with hip pain.

This study aims to conduct a synthesis of qualitative evidence, guided by the CSM, to explore middle-aged and older adults' views, beliefs, expectations and preferences about their chronic hip pain and its care, across different healthcare settings and contexts. We will use the CSM to understand how people with chronic hip pain draw on their beliefs, preferences and expectations to select coping strategies (such as seeking healthcare or choosing treatments), to judge how well their coping strategies address their wants and needs and to decide whether to maintain or modify their coping strategies based on these judgements.

\section{METHODS}

This protocol has been registered on PROSPERO and is available at http://wwwcrdyorkacuk/PROSPERO. Any important amendments to this protocol will be updated on PROSPERO. This protocol is reported according to the Preferred Reporting Items for Systematic Reviews and Meta-Analyses (PRISMA) Statement for Protocols (PRISMA-P) checklist and where relevant the Enhancing Transparency in Reporting the synthesis of Qualitative research (ENTREQ) checklist (see online supplemental appendix 1). ${ }^{69} 70$ Guided by the Cochrane Qualitative and Implementation Methods Group, ${ }^{51}$ we plan to perform a qualitative evidence synthesis using a framework synthesis methodology. ${ }^{71}$ Our qualitative evidence synthesis will 
be reported per the PRISMA Statement ${ }^{73}$ and ENTREQ ${ }^{70}$ guidelines.

\section{Eligibility criteria}

We will include data from primary studies that use qualitative methods for both data collection and data analysis and are published in peer-reviewed journals. We will exclude studies that collect data with qualitative methods but use quantitative methods for analysis. Articles will be included if they describe middle-aged and/or older adults' lived experiences, attitudes, views, beliefs, values, preferences and/or expectations about their chronic hip pain and/or its care. We define chronic hip pain: either osteoarthritis and/or femoroacetabular impingement and/or gluteal tendinopathy (also greater trochanter pain syndrome and lateral hip pain), and/or pain in the hip/groin/buttock region reported for greater than 3 months, and/or any definition of 'chronic hip pain' used by the primary study authors. ${ }^{74}$ Studies will be excluded if they describe a sample with a mean age of under 45 years or if $80 \%$ of study participants are under the age of 45 years and data about participants older than 45 years of age are not reported separately. We acknowledge there is no accepted age at which middle-age commences; however, 45 years has been used as the lower limit of middle age by the Royal Australia College of General Practitioners in a clinical guideline. ${ }^{75}$ Mixed-methods studies will be included if the qualitative data are reported separately. Studies involving participant samples with mixed pain sites, concurrent pain sites and/or other chronic conditions will be included if the data relating to participants with hip pain are reported separately, or if more than $80 \%$ of the participants in the study have chronic hip pain. Studies reporting systemic conditions (eg, rheumatoid arthritis, psoriatic arthritis or ankylosing spondylitis) will be excluded as recommended care for these conditions differs from chronic hip pain of non-systemic origin. ${ }^{76-78}$ Only English language reports will be included.

\section{Search strategy}

Following recommendations by Cochrane Qualitative and Implementation Methods Group, we will use a comprehensive search strategy-appropriate for our chosen methodology of a framework synthesis. ${ }^{7179}$ To maximise the retrieval of eligible studies, we will adapt a search strategy of patient views and preferences. ${ }^{80}$ An academic librarian from The University of Melbourne will assist to search five electronic databases including MEDLINE, CINAHL, The Cochrane Central Register of Controlled Trials, EMBASE and PsycINFO. These databases were selected to ensure a comprehensive retrieval of relevant studies and they are recommended for health topic searches. ${ }^{7981}$ Qualitative search filters will then be applied to improve the specificity of the search strategy. ${ }^{79} 81$ The search strategy will combine qualitative search filters, key search terms, and indexed terms with Boolean Operators relating to qualitative research, chronic hip pain, common diagnoses of chronic hip pain and patient perspective (see online supplemental appendix 2). We will not apply any limits on the publication date to explore potential changes in peoples' views and beliefs about chronic hip pain over time. We will manually check the reference lists of all included studies. We will search the citation lists of eligible studies using Google Scholar and Web of Science and screen these studies per our aforementioned processes. $^{79} 81$

\section{Data management}

One researcher will perform the initial search and upload the search results from all databases to EndNote reference manager (EndnoteX V.9, Clarivate Analytics, Boston; available at http://endnote.com/). Search results will then be transferred to Covidence systematic review software (Veritas Health Innovation, Melbourne, Australia; available at www.covidence.org) for study screening and to remove duplicates. Study selection will be completed using Covidence.

\section{Study selection}

Two reviewers will first independently assess the titles and abstracts of all identified studies using a priori inclusion and exclusion criteria to determine their potential eligibility. We will then obtain the full text of studies deemed potentially eligible by at least one reviewer. Two reviewers will then independently apply a priori inclusion and exclusion criteria to the full texts. Studies deemed eligible by both reviewers will be included in the review. Any disagreements between reviewers will be resolved through consensus with a third author. We will use a PRISMA flow diagram to document our searching, screening and selecting of studies for inclusion. ${ }^{73}$

Two reviewers will independently read through the primary studies to immerse themselves in the data and then independently use a prepiloted electronic data collection form to conduct data extraction. Data extracted from the articles will include: (1) location and setting, (2) sample characteristics, (3) research question, aims, sample recruitment, inclusion and exclusion criteria, methods of data collection and analysis, (4) theoretical frameworks used in data collection or analysis and (5) findings (participant quotes, second-order themes or subthemes or authors' analyses and interpretations, conclusions and recommendations).

The domains of the CSM will guide us with extracting study findings relating to middle-aged or older adults' lived experiences, views, beliefs, expectations and preferences about their chronic hip pain and/or its care. ${ }^{5758}$ Data (participant quotes, second-order themes or subthemes or authors' analyses and interpretations, conclusions and recommendations) will be extracted and deductively coded under the CSM domain ${ }^{5758}$ : (1) identity beliefs: a label and/or symptoms of hip pain or experience of care received for hip pain, (2) time-line beliefs: expected duration of hip pain and temporal nature of hip pain, (3) consequence beliefs: what is the physical, psychological, social and cognitive impact, (4) 
cause beliefs: what causes the symptoms and flare-ups, (5) control beliefs: how well can one control their hip pain or expectations about care for their hip pain. The CSM was selected using the Behaviour of Interest-Health Condition or Setting-Exclusions-Models or Theories tool, recommended by the Cochrane Qualitative and Implementation Methods Group. ${ }^{54} 82$ The CSM is relevant to our review aims, has empirical support, ${ }^{61-67}$ and there is experience within our review team with using the CSM in qualitative studies. 84768

\section{Data synthesis}

We plan to synthesise the extracted data using a framework synthesis methodology guided by the CSM. ${ }^{71} 72$ We chose a framework synthesis as this approach allows us to deductively compare our data with an empirically supported framework while enabling us to inductively refine our chosen framework (CSM) to inform clinical practice. A framework synthesis uses an a priori framework to guide a largely deductive and structured approach to data extraction and synthesis. ${ }^{72}$ However, further inductive thematic analyses may be appropriate for data that do not map to the domains of the CSM or for generating subthemes within the domains of the CSM. ${ }^{5272}$ We also chose a framework synthesis as it accommodates varying types of qualitative studies and generates review findings that are relevant to clinicians, researchers and policymakers. $^{71}$

On completing data extraction according to the domains of the CSM, two independent reviewers will then compare and discuss their coding of the data, to ensure that their analysis is supported by the findings of the primary studies and is consistent with our review aims. The reviewers will seek to identify and explain consistencies and relationships within and between the domains of the CSM. Attention will also be paid to any inconsistencies in the data that challenge our interpretations. ${ }^{83}$ The data synthesis will be an iterative process of moving between the data, the domains of the CSM, emerging subthemes and the original studies. ${ }^{83}$ If appropriate, the domains of the CSM or subthemes may be condensed or refined if better supported by the data.

Data synthesis will continue until consensus is reached on which a priori domains of the CSM are supported by the data-potentially including subthemes-and if appropriate, what new themes are generated outside of the CSM. This will be guided by discussions within the review team. The findings of the framework synthesis will be described in text and tables. A diagram may be created mapping the themes, subthemes (if generated) and the relationships between them. If we identify relationships between participant characteristics and differences within, or between, themes, we will report this in our review findings.

\section{Study quality assessment}

Two authors will independently use the Critical Appraisal Skills Programme (CASP) qualitative studies checklist to assess study methodological strengths and limitations. ${ }^{84}$ Disagreements will be resolved through consensus with a third author. The CASP qualitative study checklist consists of 10 questions and maps to recommendations made by the Cochrane Qualitative and Implementation Methods Group for assessing study strengths and limitations. ${ }^{51}$ The CASP qualitative study checklist assesses ${ }^{84}$ :

- Was there a clear statement of the aims of the research?

- Is a qualitative methodology appropriate?

- Was the research design appropriate to address the aims of the research?

- Was the recruitment strategy appropriate to the aims of the research?

- Was the data collected in a way that addressed the research issue?

- Has the relationship between researcher and participants been adequately considered?

- Have ethical issues been taken into consideration?

- Was the data analysis sufficiently rigorous?

- Is there a clear statement of findings?

- How valuable is the research?

We will create a summary table detailing all findings relating to each question of the CASP qualitative studies checklist as listed above. A narrative summary of the methodological strengths and limitations of each study will be produced. Consistent with recommendations, neither total quality scores nor cut-off points for study inclusion or exclusion will be used. ${ }^{51}$

\section{Evidence quality assessment}

At least two reviewers will use the Grading of Recommendations Assessment, Development and Evaluation-Confidence in the Evidence from Reviews of Qualitative research (GRADE-CERQual), as recommended by the Cochrane Qualitative and Implementation Methods Group, ${ }^{51}$ to assess our confidence in our individual review findings. ${ }^{51} 85$ Individual review findings are the output of qualitative evidence synthesis (eg, themes and subthemes). ${ }^{50}$ The GRADE-CERQual approach has four components:

1. Methodological limitations: Are there problems in the design or conduct in the primary studies contributing evidence to a review finding?

2. Relevance: Do the primary studies contributing evidence to a review finding apply to the context specified in the review question (eg, population, review question and setting)?

3. Coherence: Is a review finding well supported by data from the contributing primary studies; are the patterns identified across the data from the primary studies clearly and convincingly explained by the review finding?

4. Adequacy: The degree of quantity and richness (do the data inform our understanding of the phenomenon of interest) of the data supporting a review finding.

To determine the final level of confidence for a review finding, we will first assess the extent to which each GRADE-CERQual component applies to the finding and 


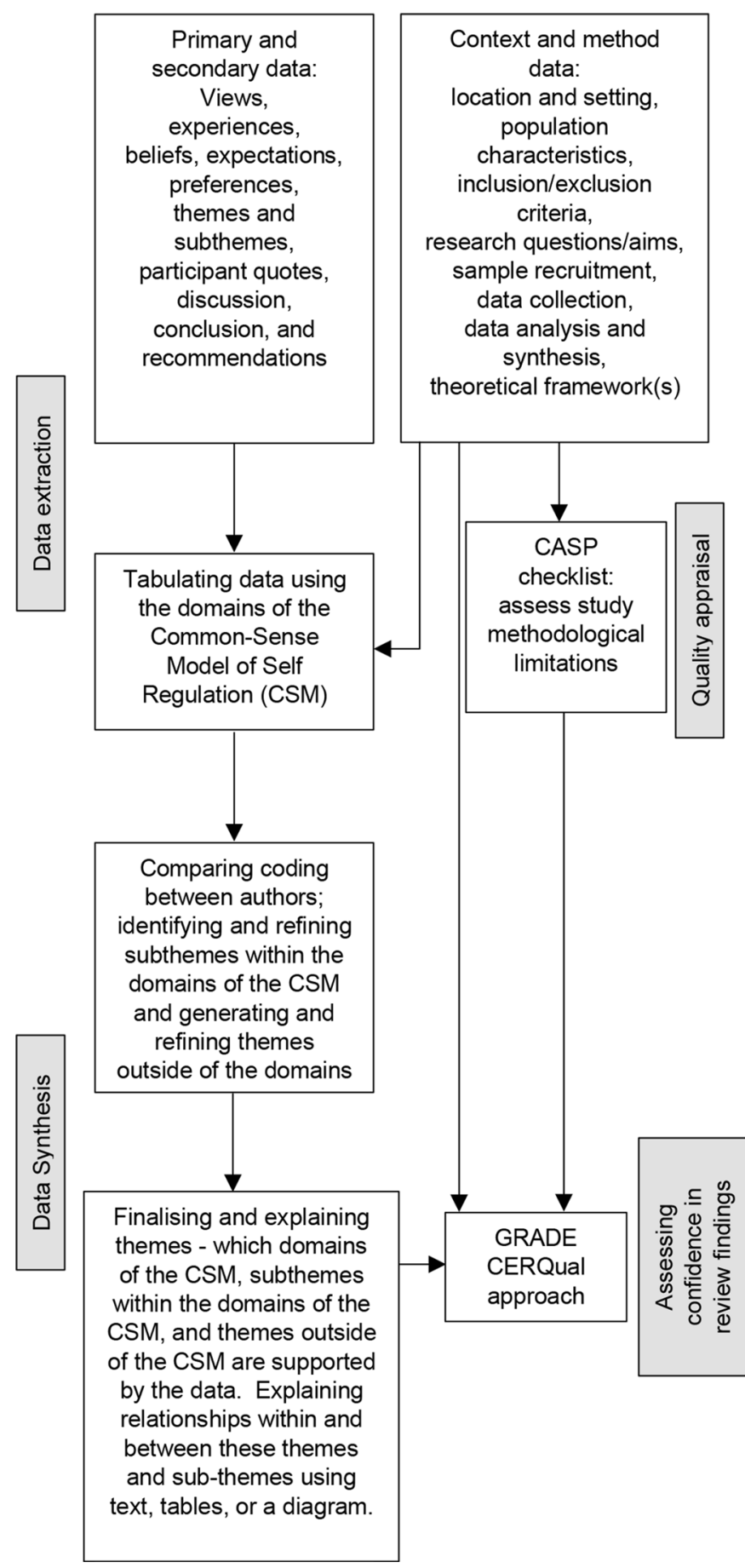

Figure 1 Flow diagram of the processes for our planned qualitative evidence synthesis using a framework approach. Methods were informed by the Cochrane Review Methods and GRADE CERQual approach. CASP, Critical Appraisal Skills Programme; GRADE CERQual, Grading of Recommendations Assessment, Development and Evaluation-Confidence in the Evidence from Reviews of Qualitative research.

then judge across all four components. ${ }^{50}$ This will be an iterative process facilitated by discussions within the review team. Confidence will be rated as high, moderate, low or very low. ${ }^{50}$ Review findings will be rated down from an initial rating of high confidence based on concerns regarding the GRADE-CERQual components. ${ }^{50}$

We will report our review findings and our GRADECERQual assessments in tables for clarity and transparency. ${ }^{50}$ A GRADE-CERQual Evidence Profile table will include summaries of the review findings, our judgements for each of the GRADE-CERQual components, our overall assessments of confidence for each review finding, our explanations for the overall GRADE-CERQual assessment and references of each of the studies contributing to a review finding. ${ }^{85}$ A Summary of Qualitative Findings table will be used to summarise review findings, the overall GRADE-CERQual assessment, explanations for each GRADE-CERQual assessment and references contributing to each review finding. ${ }^{50} 85$ Our planned procedure for data extraction through to evidence quality assessment is shown in figure 1.

\section{Patient and public involvement}

Patients and the public will not be directly involved in the design or the conduct of the review. We have discussed our study aims with people with chronic hip pain to ensure that our study is relevant to people who live with chronic hip pain.

\section{Ethics and dissemination}

Ethical approval is not required for this systematic review as primary data will not be collected. The findings of the review will be disseminated through publication in an academic journal and scientific conferences.

\section{DISCUSSION}

A shift to patient-centred care for people with musculoskeletal pain is needed. ${ }^{39} 4086$ People with chronic hip pain experience considerable physical and social disability ${ }^{1-4}$; their use of, and adherence to, recommended treatments are low, such as exercise and weight loss, and dissatisfaction with care is common. ${ }^{8} 17-2042$ This is influenced by people's views, beliefs and expectations about their joint pain and its care. ${ }^{8184345474868}$

People draw on their beliefs about joint pain when choosing coping strategies, such as seeking healthcare or selecting treatments. ${ }^{854768}$ Unhelpful beliefs about joint pain (eg, expecting little benefit from treatments) can deter people from using recommended treatments such as exercise. ${ }^{8} 454748$ Dissatisfactory healthcarehealthcare failing to address patient expectations, wants and/or needs-reinforces unhelpful beliefs about joint pain, further deterring people from using recommended treatments. ${ }^{8184245}$ To improve healthcare use, treatment adherence and patient satisfaction for people with chronic hip pain, we need a comprehensive understanding of how people with hip pain view their health problem and its care.

To our knowledge, our study will be the first qualitative evidence synthesis of middle-aged and older adults' views, beliefs, expectations and preferences about their 
chronic hip pain and its care. Qualitative methods best explore consumer views and experiences; qualitative evidence synthesis brings together evidence about consumer views to inform the design and delivery of healthcare. ${ }^{7187}$ Our qualitative evidence synthesis will use a framework approach, ${ }^{72}$ guided by the CSM for data extraction and synthesis. ${ }^{57} 58$ The CSM provides a framework to understand how people's beliefs influence their choice of, and adherence to, treatments and coping strategies. ${ }^{57-59}$

A strength of our review will be its systematic approach informed by the Cochrane Qualitative and Implementation Methods Group, ${ }^{5179}$ including our plan to report methodological strengths and limitations of each included study ${ }^{84}$ and to provide ratings of confidence for each review finding. ${ }^{50}$ Our review will be limited by excluding studies published in languages other than English, which may limit the transferability of our findings to non-English-speaking countries, high-income countries or minorities. To help the reader interpret the transferability of our findings, where possible, we will report the location, race, culture, language and ethnicity of the study participants informing our review findings. Our study is further limited by only extracting published data. Hence, we may miss details or meaning present in the unpublished data and we acknowledge that the data we extract for synthesis have already been interpreted by the authors of the primary studies.

We anticipate that our review findings will enhance our understanding of middle-aged and older adults' views, beliefs, expectations, and preferences about their chronic hip pain and its care, across different healthcare settings and contexts. By using the CSM, we will explore how people with chronic hip pain draw on their beliefs, preferences and expectations to choose coping strategies, to judge how well their coping strategies address their wants and needs and to decide whether to maintain or modify their coping strategies based on these judgements. These findings, by informing patientcentred approaches to care, can drive better healthcare use, adherence to treatments, patient satisfaction and clinical outcomes for middle-aged and older adults with chronic hip pain.

\section{Twitter Travis Haber @haber_travis}

Contributors $\mathrm{TH}, \mathrm{MH}, \mathrm{RSH}, \mathrm{FD}$ and SB planned and designed the study and protocol. TH and MH drafted the protocol manuscript with input from RSH, FD and SB. All authors have read and approved the final manuscript. TH and MH guarantee the paper and that the authorship statement is correct.

Funding RSH is funded by an NHMRC Senior Research Fellowship (APP1152217). $\mathrm{MH}$ is funded by am NHMRC Investigator Grant Emerging Leader 1 (APP1172928).

Competing interests None declared.

Patient consent for publication Not applicable.

Provenance and peer review Not commissioned; externally peer reviewed.

Supplemental material This content has been supplied by the author(s). It has not been vetted by BMJ Publishing Group Limited (BMJ) and may not have been peer-reviewed. Any opinions or recommendations discussed are solely those of the author(s) and are not endorsed by BMJ. BMJ disclaims all liability and responsibility arising from any reliance placed on the content. Where the content includes any translated material, BMJ does not warrant the accuracy and reliability of the translations (including but not limited to local regulations, clinical guidelines, terminology, drug names and drug dosages), and is not responsible for any error and/or omissions arising from translation and adaptation or otherwise.

Open access This is an open access article distributed in accordance with the Creative Commons Attribution Non Commercial (CC BY-NC 4.0) license, which permits others to distribute, remix, adapt, build upon this work non-commercially, and license their derivative works on different terms, provided the original work is properly cited, appropriate credit is given, any changes made indicated, and the use is non-commercial. See: http://creativecommons.org/licenses/by-nc/4.0/.

\section{ORCID iDs}

Travis Haber http://orcid.org/0000-0001-5487-0851

Michelle Hall http://orcid.org/0000-0003-2024-4945

\section{REFERENCES}

1 Plinsinga ML, Coombes BK, Mellor R, et al. Psychological factors not strength deficits are associated with severity of gluteal tendinopathy: a cross-sectional study. Eur J Pain 2018;22:1124-33.

2 Griffin DR, Dickenson EJ, O'Donnell J, et al. The warwick agreement on femoroacetabular impingement syndrome (FAI syndrome): an international consensus statement. Br J Sports Med 2016;50:1169-76.

3 Segal NA, Felson DT, Torner JC, et al. Greater trochanteric pain syndrome: epidemiology and associated factors. Arch Phys Med Rehabil 2007;88:988-92.

4 Cross M, Smith E, Hoy D, et al. The global burden of hip and knee osteoarthritis: estimates from the global burden of disease 2010 study. Ann Rheum Dis 2014;73:1323-30.

5 Christmas C, Crespo CJ, Franckowiak SC. How common is hip pain among older adults. J Fam Pract 2002;51:346-8.

6 Murphy LB, Helmick CG, Schwartz TA, et al. One in four people may develop symptomatic hip osteoarthritis in his or her lifetime. Osteoarthritis Cartilage 2010;18:1372-9.

7 Sundén-Lundius A, Johnsson B, Lohmander S, et al. Prevalence of self-reported hip disorders, relations to age, gender, pain, stiffness, weakness and other joint disorders. Adv Physiother 2005;7:108-13.

8 I R de Oliveira B, Smith AJ, O'Sullivan PPB, et al. 'My hip is damaged': a qualitative investigation of people seeking care for persistent hip pain. Br J Sports Med 2020;54:858-65.

9 Metcalfe D, Perry DC, Claireaux HA. Does this patient have hip osteoarthritis?: the rational clinical examination systematic review. JAMA 2019;322:2323-33.

10 Grimaldi A, Mellor R, Hodges P, et al. Gluteal tendinopathy: a review of mechanisms, assessment and management. Sports Med 2015;45:1107-19.

11 Reiman MP, Agricola R, Kemp JL, et al. Consensus recommendations on the classification, definition and diagnostic criteria of hip-related pain in young and middle-aged active adults from the international hip-related pain research network, Zurich 2018. Br J Sports Med 2020;54:631-41.

12 Woodley SJ, Nicholson HD, Livingstone V, et al. Lateral hip pain: findings from magnetic resonance imaging and clinical examination. J Orthop Sports Phys Ther 2008;38:313-28.

13 Bannuru RR, Osani MC, Vaysbrot EE, et al. OARSI guidelines for the non-surgical management of knee, hip, and polyarticular osteoarthritis. Osteoarthritis Cartilage 2019;27:1578-89.

14 National Institute for Health and Care Excellence (GB). Osteoarthritis: care and management in adults. London: National Institute for Health and Care Excellence, 2014.

15 Katz JN, Arant KR, Loeser RF. Diagnosis and treatment of hip and knee osteoarthritis: a review. JAMA 2021;325:568-78.

16 Kolasinski SL, Neogi T, Hochberg MC, et al. 2019 American college of rheumatology/arthritis foundation guideline for the management of osteoarthritis of the hand, hip, and knee. Arthritis Rheumatol 2020;72:220-33.

17 Hinman RS, Nicolson PJA, Dobson FL, et al. Use of nondrug, nonoperative interventions by community-dwelling people with hip and knee osteoarthritis. Arthritis Care Res 2015;67:305-9.

18 Papandony MC, Chou L, Seneviwickrama M, et al. Patients perceived health service needs for osteoarthritis (OA) care: a scoping systematic review. Osteoarthritis Cartilage 2017;25:1010-25.

19 Brand CA, Harrison C, Tropea J, et al. Management of osteoarthritis in general practice in Australia. Arthritis Care Res 2014;66:551-8.

20 Young JL, Wright AA, Rhon DI. Nonoperative management prior to hip arthroscopy for femoroacetabular impingement syndrome: an 
investigation into the utilization and content of physical therapy. $J$ Orthop Sports Phys Ther 2019;49:593-600.

21 National Clinical Guideline Centre (GB). Patient experience in adult NHS services: improving the experience of care for people using adult NHS services: patient experience in generic terms. London: Royal College of Physicians, 2012.

22 Minister of Health (NZ). New Zealand health strategy: future direction. Wellington: Ministry of Health, 2016.

23 Paparella G. Person-centred care in Europe: a cross-country comparison of health system performance, strategies and structures. Picker Institute: Oxford, 2016.

24 Australian Commission on Safety and Quality in Health Care (AU). Patient-centred care: improving quality and safety through partnerships with patients and consumers. Sydney: Australian Commission on Safety and Quality in Health Care, 2011.

25 World Health Organization (CH). WHO global strategy on peoplecentred and integrated health services: interim report. Geneva: World Health Organization, 2015

26 Frampton G, Guastello S, Brady C. Patient-centred care improvement guide. Derby: Planetree, Inc. and Picker Institute, 2008.

27 Beach MC, Sugarman J, Johnson RL, et al. Do patients treated with dignity report higher satisfaction, adherence, and receipt of preventive care? Ann Fam Med 2005;3:331-8.

28 DiGioia A, Greenhouse PK, Levison TJ. Patient and family-centered collaborative care: an orthopaedic model. Clin Orthop Relat Res 2007;463:13-19.

29 Isaac T, Zaslavsky AM, Cleary PD, et al. The relationship between patients' perception of care and measures of hospital quality and safety. Health Serv Res 2010;45:1024-40.

30 Jha AK, Orav EJ, Zheng J, et al. Patients' perception of hospital care in the United States. N Engl J Med 2008;359:1921-31.

31 Tsai TC, Orav EJ, Jha AK. Patient satisfaction and quality of surgical care in US hospitals. Ann Surg 2015;261:2-8.

32 Ruben MA, Meterko M, Bokhour BG. Do patient perceptions of provider communication relate to experiences of physical pain? Patient Educ Couns 2018;101:209-13.

33 Pinto RZ, Ferreira ML, Oliveira VC, et al. Patient-centred communication is associated with positive therapeutic alliance: a systematic review. J Physiother 2012;58:77-87.

34 Little $\mathrm{P}$, Everitt $\mathrm{H}$, Williamson I, et al. Observational study of effect of patient centredness and positive approach on outcomes of general practice consultations. BMJ 2001;323:908-11.

35 World Health Organisation (CH). People-centred and integrated health services: an overview of the evidence: interim report. Geneva: World Health Organisation, 2015.

36 Coulter A. Do patients want a choice and does it work? BMJ 2010;341:c4989.

37 Coulter A, Entwistle VA, Eccles A. Personalised care planning for adults with chronic or long-term health conditions. Cochrane Database Syst Rev 2015;3:CD010523.

38 Dwamena F, Holmes-Rovner M, Gaulden CM, et al. Interventions for providers to promote a patient-centred approach in clinical consultations. Cochrane Database Syst Rev 2012;12:CD003267.

39 Lin I, Wiles L, Waller R. Patient-centred care: the cornerstone for high-value musculoskeletal pain management. Br J Sports Med 2020;54:1240-2.

40 Lin I, Wiles L, Waller R, et al. What does best practice care for musculoskeletal pain look like? Eleven consistent recommendations from high-quality clinical practice guidelines: systematic review. $\mathrm{Br} J$ Sports Med 2020;54:79-86.

41 Chen J, Hu F, Yang BX, et al. Experience of living with pain among older adults with arthritis: a systematic review and meta-synthesis. Int J Nurs Stud 2020;111:103756.

42 Chou L, Ellis L, Papandony M, et al. Patients' perceived needs of osteoarthritis health information: a systematic scoping review. PLOS One 2018;13:e0195489.

43 Wallis JA, Taylor NF, Bunzli S, et al. Experience of living with knee osteoarthritis: a systematic review of qualitative studies. BMJ Open 2019;9:e030060.

44 Wride JM, Bannigan K. 'If you can't help me, so help me god I will cut it off myself...' the experience of living with knee pain: a qualitative meta-synthesis. Physiotherapy 2018;104:299-310.

45 Hurley M, Dickson K, Hallett R, et al. Exercise interventions and patient beliefs for people with hip, knee or hip and knee osteoarthritis: a mixed methods review. Cochrane Database Syst Rev 2018;4:CD010842

46 Smith TO, Purdy R, Lister S, et al. Attitudes of people with osteoarthritis towards their conservative management: a systematic review and meta-ethnography. Rheumatol Int 2014;34:299-313.

47 Bunzli S, O'Brien P, Ayton D, et al. Misconceptions and the acceptance of evidence-based nonsurgical interventions for knee osteoarthritis. A qualitative study.. Clin Orthop Relat Res 2019;477:1975-83.

48 Darlow B, Brown M, Thompson B, et al. Living with osteoarthritis is a balancing act: an exploration of patients' beliefs about knee pain. BMC Rheumatol 2018;2:1-9.

49 Smith TO, Purdy R, Lister S, et al. Living with osteoarthritis: a systematic review and meta-ethnography. Scand J Rheumatol 2014;43:441-52.

50 Lewin S, Glenton C, Munthe-Kaas $\mathrm{H}$, et al. Using qualitative evidence in decision making for health and social interventions: an approach to assess confidence in findings from qualitative evidence syntheses (GRADE-CERQual). PLoS Med 2015;12:e1001895.

51 Noyes J, Booth A, Flemming K, et al. Cochrane qualitative and implementation methods group guidance series-paper 3: methods for assessing methodological limitations, data extraction and synthesis, and confidence in synthesized qualitative findings. J Clin Epidemiol 2018;97:49-58.

52 Carroll C, Booth A, Leaviss J, et al. "Best fit" framework synthesis: refining the method. BMC Med Res Methodol 2013;13:37.

53 Anderson LM, Oliver SR, Michie S, et al. Investigating complexity in systematic reviews of interventions by using a spectrum of methods. $J$ Clin Epidemiol 2013;66:1223-9.

54 Noyes J, Hendry M, Booth A, et al. Current use was established and cochrane guidance on selection of social theories for systematic reviews of complex interventions was developed. J Clin Epidemiol 2016;75:78-92.

55 Michie S, West R, Campbell R. ABC of behaviour change theories. Sutton: Silverback publishing, 2014: 457-63.

56 Davis R, Campbell R, Hildon Z, et al. Theories of behaviour and behaviour change across the social and behavioural sciences: a scoping review. Health Psychol Rev 2015;9:323-44.

57 Leventhal H, Diefenbach M, Leventhal EA. Illness cognition: using common sense to understand treatment adherence and affect cognition interactions. Cognit Ther Res 1992;16:143-63.

58 Leventhal H, Phillips LA, Burns E. The common-sense model of self-regulation (CSM): a dynamic framework for understanding illness self-management. J Behav Med 2016;39:935-46.

59 Hagger MS, Orbell S. The common sense model of illness selfregulation: a conceptual review and proposed extended model. Health Psychol Rev 2021:1-31.

60 Leventhal $\mathrm{H}$. Next steps for examining the common-sense of health behaviour. Health Psychol Rev 2019;13:487-9.

61 Broadbent $\mathrm{E}$, Wilkes $\mathrm{C}$, Koschwanez $\mathrm{H}$, et al. A systematic review and meta-analysis of the brief illness perception questionnaire. Psychol Health 2015;30:1361-85.

62 Dempster M, Howell D, McCorry NK. Illness perceptions and coping in physical health conditions: a meta-analysis. J Psychosom Res 2015;79:506-13.

63 Hagger MS, Koch S, Chatzisarantis NLD, et al. The common sense model of self-regulation: meta-analysis and test of a process model. Psychol Bull 2017;143:1117-54

64 Appelt CJ, Burant CJ, Siminoff LA, et al. Arthritis-specific health beliefs related to aging among older male patients with knee and/or hip osteoarthritis. J Gerontol A Biol Sci Med Sci 2007;62:184-90.

65 Botha-Scheepers S, Riyazi N, Kroon HM, et al. Activity limitations in the lower extremities in patients with osteoarthritis: the modifying effects of illness perceptions and mental health. Osteoarthritis Cartilage 2006;14:1104-10.

66 Kaptein AA, Bijsterbosch J, Scharloo M, et al. Using the common sense model of illness perceptions to examine osteoarthritis change: a 6-year longitudinal study. Health Psychol 2010;29:56-64.

67 Orbell S, Johnston M, Rowley D, et al. Cognitive representations of illness and functional and affective adjustment following surgery for osteoarthritis. Soc Sci Med 1998;47:93-102.

68 Bunzli S, Smith A, Schütze R, et al. Making sense of low back pain and pain-related fear. J Orthop Sports Phys Ther 2017;47:628-36.

69 Moher D, Shamseer L, Clarke M, et al. Preferred reporting items for systematic review and meta-analysis protocols (PRISMA-P) 2015 statement. Syst Rev 2015;4:1-9.

70 Tong A, Flemming K, Mclnnes E, et al. Enhancing transparency in reporting the synthesis of qualitative research: ENTREQ. BMC Med Res Methodol 2012;12:181.

71 Booth A, Noyes J, Flemming K. Guidance on choosing qualitative evidence synthesis methods for use in health technology assessments of complex interventions. Available: http://www. integrate-hta.eu/downloads/ [Accessed Jan 2021].

72 Barnett-Page E, Thomas J. Methods for the synthesis of qualitative research: a critical review. BMC Med Res Methodol 2009;9:1-11.

73 Moher D, Liberati A, Tetzlaff J, et al. Preferred reporting items for systematic reviews and meta-analyses: the PRISMA statement. BMJ 2009;339:b2535. 
74 Nicolson PJA, Bennell KL, Dobson FL, et al. Interventions to increase adherence to therapeutic exercise in older adults with low back pain and/or hip/knee osteoarthritis: a systematic review and metaanalysis. Br J Sports Med 2017:51:791-9.

75 The Royal Australian College of General Practitioners (AU). Guidelines for preventive activities in general practice. 9th edn. East Melbourne: RACGP, 2018.

76 Ward MM, Deodhar A, Gensler LS. Update of the american college of rheumatology/spondylitis association of america/spondyloarthritis research and treatment network recommendations for the treatment of ankylosing spondylits and nonradiographic axial spondyloarthritis. Arthritis Rheumatol 2019;2019:1599-613.

77 Singh JA, Guyatt G, Ogdie A. American college of rheumatology/ national psoriasis foundation guideline for the treatment of psoriatic arthritis. Arthritis Rheumatol 2018;2019:5-32.

78 Sammaritano LR, Bermas BL, Chakravarty EE. American college of rheumatology guideline for the management of reproductive health in rheumatic and musculoskeletal diseases. Arthritis Rheumatol 2020;2020:529-56.

79 Harris JL, Booth A, Cargo M, et al. Cochrane qualitative and implementation methods group guidance series-paper 2: methods for question formulation, searching, and protocol development for qualitative evidence synthesis. J Clin Epidemiol 2018;97:39-48.

80 Selva A, Solà I, Zhang Y, et al. Development and use of a content search strategy for retrieving studies on patients' views and preferences. Health Qual Life Outcomes 2017;15:1-9.
81 Booth A. Searching for qualitative research for inclusion in systematic reviews: a structured methodological review. Syst Rev 2016;5:1-23.

82 Booth A, Carroll C. Systematic searching for theory to inform systematic reviews: is it feasible? Is it desirable? Health Info Libr J 2015;32:220-35.

83 Booth A, Sutton A, Papaioannou D. Systematic approaches to a successful literature review. London: Sage Publications Ltd, 2016: 215-71.

84 Critical Appraisal Skills Programme (CASP). CASP qualitative research checklist: 10 questions to help you make sense of qualitative research. Available: https://casp-uk.b-cdn.net/wpcontent/uploads/2018/03/CASP-Qualitative-Checklist-2018_fillable form.pdf [Accessed Feb 2021].

85 Lewin S, Booth A, Glenton C, et al. Applying GRADE-CERQual to qualitative evidence synthesis findings: introduction to the series. Implement Sci 2018;13:1-10.

86 Caneiro JP, Roos EM, Barton CJ, et al. It is time to move beyond 'body region silos' to manage musculoskeletal pain: five actions to change clinical practice. Br J Sports Med 2020;54:438-9.

87 Glenton C, Lewin S, Norris S. Chapter 15: using evidence from qualitative research to develop WHO guidelines. In: Norris S, ed. World health organization handbook for guideline development. 2nd edn. Geneva: WHO, 2016: 183-200. 